\title{
Prevention of relapse in patients with congestive heart failure: the role of precipitating factors
}

\author{
J Feenstra, D E Grobbee, F A M Jonkman, A W Hoes, B H Ch Stricker
}

Department of

Epidemiology and

Biostatistics,

Pharmacoepidemiology

Unit, Erasmus

University Medical

School, Rotterdam,

Netherlands

J Feenstra

D E Grobbee

B H Ch Stricker

Department of

Cardiology, Thorax

Center Dijkzigt,

Erasmus University

Medical School

F A M Jonkman

Inspectorate for Health Care, Drug Safety

Unit, Rijswijk,

Netherlands

J Feenstra

B H Ch Stricker

Julius Center for

Patient-Oriented

Research, Utrecht

University Academic

Hospital, Utrecht,

Netherlands

D E Grobbee

A W Hoes

Correspondence to: Dr B H Ch Stricker,

Department of Epidemiology and Biostatistics, Erasmus University Medical School, Dr Molewaterplein 503000 DR Rotterdam, Netherlands. email: stricker@

epib.fgg.eur.nl

Accepted for publication 2 July 1998

\begin{abstract}
Relapse of congestive heart failure (CHF) frequently occurs and has serious consequences in terms of morbidity, mortality, and health care expenditure. Many studies have investigated the aetiological and prognostic factors of CHF, but there are only limited data on the role of precipitating factors that trigger relapse of $\mathrm{CHF}$. Knowledge of potential precipitating factors may help to optimise treatment and provide guidance for patients with $\mathrm{CHF}$. The literature was reviewed to identify factors that may influence haemodynamic homeostasis in CHF. Precipitating factors that may offer opportunities for preventing relapse of CHF were selected. Potential precipitating factors are discussed in relation to the pathophysiology of $\mathrm{CHF}$ : alcohol, smoking, psychological stress, uncontrolled hypertension, cardiac arrhythmias, myocardial ischaemia, poor treatment compliance, and inappropriate medical treatment. Poor treatment compliance in particular is frequently encountered in patients with CHF. Furthermore, studies of medical treatment under everyday circumstances indicate that some aspects of the management of $\mathrm{CHF}$ can be improved. In conclusion, the identification of precipitating factors for relapse of CHF may strongly contribute to optimal treatment. Improvement of treatment compliance and optimalisation of medical treatment may offer important possibilities to clinicians to reduce the number of relapses in patients with $\mathbf{C H F}$.

(Heart 1998;80:432-436)
\end{abstract}

Keywords: congestive heart failure; precipitating factors; prevention

There is increasing interest in congestive heart failure $(\mathrm{CHF})$ from both clinicians and researchers. The prevalence of $\mathrm{CHF}$ continues to increase despite advances in the treatment of various risk factors for this disease, such as hypertension and coronary artery disease. ${ }^{1}$ This increase is the result of several medical and demographic developments: an aging population, decreasing mortality of patients with acute myocardial infarction, and im- proved treatment of patients with angina pectoris and hypertension. ${ }^{2}$ In addition, survival in patients with $\mathrm{CHF}$ has improved since the introduction of angiotensin converting enzyme (ACE) inhibitors.

$\mathrm{CHF}$ is clinically characterised by periods of remission and exacerbation. Readmission rates of up to $25 \%$ within six months after a previous hospital discharge for $\mathrm{CHF}$ have been reported in patients older than 65 years. ${ }^{34}$ Relapse of $\mathrm{CHF}$ in patients with previously stable compensated heart failure may be caused by deteriorating ventricular function, but several precipitating factors have been suggested. ${ }^{56}$ Some precipitating factors can be regarded as potentially preventable. Research on precipitating factors leading to relapses of $\mathrm{CHF}$, however, is scarce. ${ }^{56}$ Nevertheless, timely identification of potential precipitating factors may offer an important advantage in efforts to reduce morbidity and the number of hospital admissions attributed to the syndrome of CHF.

We conducted a search of the MEDLINE database from 1966 to December 1997 and used lateral references to review the literature on potential precipitating factors. In this article we focus on the role of precipitating factors that are relatively common in clinical practice and potentially modifiable: the effects of alcohol, smoking, psychological stress, uncontrolled hypertension, cardiac arrhythmias, myocardial ischaemia, lack of compliance, and inappropriate medical treatment.

Precipitating factors for relapse of congestive heart failure

Knowledge of potential precipitating factors for relapse of CHF is crucial to prevent or postpone such relapses. In a descriptive study, Ghali et $a \bar{P}$ examined potential precipitating factors in 101 hospital readmissions for CHF. Precipitating factors were identified in $93 \%$ of patients. The most common factor was lack of adherence to the prescribed medical regimen $(64 \%$ of patients). Other frequently identified precipitating factors were uncontrolled hypertension (44\%), cardiac arrhythmias (29\%), iatrogenic factors (21\%), and pulmonary infection (17\%) (table 1). In a more recent study, Opasich et al identified precipitating factors in $91 \%$ of 328 instances of non-fatal decompensation in 304 patients. All patients had a history of at least one previous episode of severe decompensation. 
Table 1 Factors that may precipitate relapses in patients with pre-existing $\mathrm{CHF}$

Lack of treatment compliance

Myocardial infarction

Angina pectoris or painless myocardial ischaemia

Alcohol consumption

Cardiac arrhythmias

Inappropriate medical treatment

Infections

Anaemia

Pulmonary embolism

Thyroid disease

Pregnancy

Physical, dietary, fluid, and environmental excesses

Emotional stress

Systemic hypertension

Smoking

Common precipitating factors were cardiac arrhythmias ( $24 \%$ of patients), poor compliance $(15 \%)$, infection $(23 \%)$, angina pectoris $(14 \%)$, and iatrogenic factors (10\%). Frequency differences in specific precipitating factors reflect the different populations and designs of the two studies. In addition, the absence of a control group excludes assessment of the relative risk of these potential precipitating factors.

Several diseases may induce the syndrome of CHF. Patients in whom CHF is diagnosed should be given optimal medical care to prevent clinical worsening while maintaining quality of life. An accurate evaluation of potential precipitating factors should also be routinely performed in patients with relapse of $\mathrm{CHF}$.

The haemodynamic effects of these precipitating factors are discussed within the framework of current views on the pathophysiology of $\mathrm{CHF}$. Apart from the precipitating factors discussed here, CHF may also be precipitated by infection, anaemia, pulmonary embolism, thyroid disease, pregnancy, and physical, dietary, fluid, and environmental excesses. ${ }^{5-7}$ As most of these factors have either known causes, such as excessive salt intake and excessive intravenous fluid administration, or relatively uncommon causes, they are not discussed in further detail.

ALCOHOL

CHF resulting from alcoholic cardiomyopathy is a relatively common cause of non-ischaemic cardiomyopathy. ${ }^{8}$ The amount and duration of alcohol consumption required to induce alcoholic cardiomyopathy is not well defined, but has been estimated at five to six ounces $(1 \mathrm{oz}=28 \mathrm{ml})$ of ethanol daily for at least 10 years. Abstention from alcohol is crucial in patients with alcohol induced cardiomyopathy. ${ }^{9}$ Echocardiographic studies show that major clinical improvement and normalisation of left ventricular function can be achieved after abstention from alcohol. ${ }^{10}{ }^{11}$ Resumed alcohol intake may be a precipitating factor for relapse of CHF.

There is no convincing evidence for absolute abstention from alcohol in patients with $\mathrm{CHF}$ not induced by alcohol. In a study in which the acute effects of moderate alcohol consumption $(0.9 \mathrm{~g} / \mathrm{kg})$ in patients with New York Heart Association (NYHA) class III-IV heart failure were assessed, small but significant reductions in arterial pressure, pulmonary artery pressure, pulmonary artery wedge pressure, and systemic vascular resistance were seen after a single dose of alcohol, but CHF did not deteriorate. ${ }^{12}$ Chronic heavy alcohol consumption should be strongly discouraged in all patients with $\mathrm{CHF}$, but there are no convincing reasons to warn against incidental moderate consumption of alcohol. ${ }^{13}$ Clinical practice shows, however, that some patients with apparently non-alcoholic induced CHF may have significant improvement after complete abstention from even incidental moderate alcohol consumption. Therefore, evaluation of a period of complete alcohol abstention in patients with severe CHF may be worthwhile.

\section{SMOKING}

Several studies have been performed on the acute haemodynamic effects of smoking. Cigarette smoking increases heart rate and blood pressure, both principal determinants of myocardial oxygen consumption. ${ }^{14}$ Goldbarg et al showed in healthy individuals that the left ventricular stroke index decreased significantly at several levels of exercise after smoking, although there was no significant change at rest. ${ }^{15}$ Aronow et al investigated the effects of cigarette smoking and breathing carbon monoxide on cardiovascular haemodynamics in patients with angina. ${ }^{16}$ This study showed that an increased level of carboxyhaemoglobin had a negative inotropic effect and increased left ventricular end diastolic pressure. There was a significant decrease in the stroke index after smoking. Pentecost et al indicated that cigarette smoking tends to decrease cardiac output especially in older patients with a history of myocardial infarction. ${ }^{17}$ Nicolozakes et al investigated the effects of smoking in patients with NYHA class III CHF. ${ }^{18}$ Cardiac output remained unchanged after smoking, but other haemodynamic changes were seen. As expected, heart rate and systemic blood pressure (double product) increased substantially after smoking. In addition, there were mild increases in pulmonary artery pressure, ventricular filling pressures, and total systemic and pulmonary vascular resistance. The increased ventricular afterload probably accounts for the observed mild decrease in stroke volume. Thus smoking increases oxygen demand but decreases myocardial oxygen supply because of reduced diastolic filling time and increased carboxyhaemoglobin level. This finding has important negative consequences for myocardial oxygen supply. Patients with CHF should be strongly advised to stop smoking and informed that continued smoking can unfavourably affect CHF. It remains unclear to what extent smoking may act as a precipitating factor for relapse of $\mathrm{CHF}$.

\section{PSYCHOLOGICAL STRESS}

Mental stress in patients with ischaemic heart disease can induce transient myocardial ischaemia and transient wall motion abnormalities. ${ }^{19}$ Rozanski et al reported that wall motion abnormalities occurred in $59 \%$ of patients with coronary artery disease during mental stress. ${ }^{20} \mathrm{~A}$ drop in ejection fraction of more than $5 \%$ was seen in 
$36 \%$ of patients. Wall motion abnormalities were seen in only $8 \%$ of normal controls during mental stress. There was no clear effect on ejection fraction in the controls. Mental stress may also induce transient changes of the electrophysiological properties of the myocardium, which may sensitise the heart to life threatening ventricular arrhythmias. ${ }^{21}$ Emotional factors preceding hospitalisation for $\mathrm{CHF}$ have been reported in $49 \%$ of patients. $^{7}$ Stress induced heart failure has been described. ${ }^{22}$ Giannuzzi et al reported that psychological stress induced changes in left ventricular diastolic function in patients with idiopathic cardiomyopathy. ${ }^{19}$ The effects of mental arithmetic on these patients were compared with those on controls. The ratio of transmitral peak flow velocity in early versus late diastole significantly increased during mental arithmetic, while transmitral deceleration time greatly decreased. These findings suggest that left ventricular function is impaired during psychological stress. Neuroendocrine activation and a significant increase in arterial blood pressure may also contribute to the haemodynamic effects of psychological stress.

The full effects of psychological stress on cardiovascular function in patients with $\mathrm{CHF}$ are not known, but many of the discussed effects may be considered unfavourable.

\section{UNCONTROLLED HYPERTENSION}

Recent data from the Framingham study underscore the importance of hypertension as a major risk factor for $\mathrm{CHF}^{23}$ Hypertension may impair ventricular function by increasing afterload and impairing systolic contraction and diastolic relaxation. ${ }^{24}$ Approximately 50\% of patients with hypertension and a normal coronary angiogram have transient ST segment depression during 24 hour Holter monitoring, usually without angina pectoris. ${ }^{25}$ As there is no relation with left ventricular hypertrophy, these findings reflect a disturbed coronary microvasculature. Intervention trials have provided convincing evidence of the efficacy of hypertension treatment in reducing the incidence of $\mathrm{CHF}^{26}$ Obviously, persistent hypertension in patients with CHF will have a detrimental effect on ventricular performance. Blood pressure in patients with end stage $\mathrm{CHF}$ usually decreases because of low cardiac output. Uncontrolled hypertension despite antihypertensive treatment, defined as diastolic blood pressure of $105 \mathrm{~mm} \mathrm{Hg}$ or more, was identified in $44 \%$ of patients readmitted to hospital for $\mathrm{CHF}^{5}$ Adequate blood pressure control in patients with $\mathrm{CHF}$ is crucial because of the numerous haemodynamic effects of hypertension, although there are few data on the extent to which uncontrolled hypertension may account for relapse of $\mathrm{CHF}$.

\section{ARRHYTHMIAS}

Cardiac arrhythmias are frequently present in patients with $\mathrm{CHF}$ and are regarded as a sign of impaired left ventricular function. Atrial fibrillation is the most prevalent cardiac arrhythmia in patients with CHF. Nevertheless, its prognostic significance is controversial. There have been studies in which atrial fibrillation did not increase morbidity and mortality as well as studies in which atrial fibrillation was a marker for an increased mortality risk. ${ }^{27}{ }^{28}$ Ghali et al reported that cardiac arrhythmias, particularly atrial fibrillation, were present in $29 \%$ of patients studied and were considered to be directly responsible for relapse of $\mathrm{CHF}$ in $78 \%$ of patients. ${ }^{5}$ Cardioversion of chronic atrial fibrillation to sinus rhythm in patients with NYHA functional class I or II significantly increased cardiac output during exercise, maximum oxygen uptake, and maximal tolerated workload. ${ }^{29}$ Successful cardioversion in patients with chronic atrial fibrillation and idiopathic dilated cardiomyopathy significantly improved left ventricular ejection fraction from $32 \%$ to $53 \%$. $^{30}$ Several studies have reported on patients with severe left ventricular dysfunction and atrial fibrillation with rapid ventricular response. ${ }^{31} 32$ Left ventricular dysfunction in these patients may be completely reversed by controlling ventricular rate or restoring sinus rhythm. The unfavourable haemodynamic consequences of atrial fibrillation compared with those of sinus rhythm suggest that development of this arrhythmia may be a trigger for relapse of CHF.

\section{MYOCARDIAL ISCHAEMIA}

The detrimental effects of myocardial ischaemia on ventricular function have been well documented. ${ }^{33}$ The main cause of progressive myocardial failure is postulated as subendocardial ischaemia, even in patients with nonischaemic $\mathrm{CHF}^{34}$ Silent or symptomatic myocardial ischaemia can also be identified as part of the pathway relating other potential precipitating factors, such as psychological stress, smoking, cardiac arrhythmias, and hypertension, to relapses of $\mathrm{CHF}^{25}{ }^{35}$ Impairment of systolic and diastolic ventricular function persists from hours to days after transient myocardial ischaemia. ${ }^{36}$ Hibernating myocardium is a widely accepted concept in modern cardiology, defined as a state of persistently impaired myocardial and left ventricular function at rest because of reduced coronary blood flow. ${ }^{33}$ Hibernating myocardium can be considered as a myocardial adaptation to reduced coronary blood flow, to prevent irreversible myocardial damage. The significant improvement of left ventricular function after coronary revascularisation is mainly based on this principle. ${ }^{33}$ Although the exact frequency of hibernating myocardium is not known, Carlson et al reported hibernating myocardium in $75 \%$ of patients with unstable angina pectoris and in $25 \%$ of patients with stable angina pectoris. ${ }^{37}$ Abolition of myocardial hibernation can improve left ventricular function. ${ }^{33}$ Therefore, prevention of myocardial ischaemia may contribute to the maintenance of haemodynamic homeostasis in patients with CHF.

LACK OF TREATMENT COMPLIANCE

Non-compliance to medication and diet has repeatedly been identified as a frequent precipitating factor for admission to hospital for decompensated heart failure. ${ }^{5}{ }^{6}$ Moreover, several studies have reported approximately 50\% 
non-compliance with long term medication regimens. ${ }^{38}$ This may represent an important impediment to effective treatment of $\mathrm{CHF}$. Interestingly, physicians' personal characteristics and characteristics of their practices also influence patients' adherence to medical treatment. ${ }^{39}$ Physicians' global job satisfaction positively influences patient compliance.

Non-compliance deserves major attention from health professionals, as it may offer an opportunity to make a significant contribution to preventing relapse in patients with $\mathrm{CHF}$.

INAPPROPRIATE MEDICAL TREATMENT

Iatrogenic factors responsible for relapses of CHF were identified by Ghali et al in $21 \%$ of relapses $^{5}$ and by Opaschi et al in $10 \%$ of relapses. ${ }^{6}$ The most important causes of iatrogenic $\mathrm{CHF}$ are usually inappropriate medication and excessive intravenous fluid administration. Although not discussed in detail here, several categories of drugs, such as non-steroidal anti-inflammatory drugs, $\beta$ blockers, and antiarrhythmics, may affect cardiovascular homeostasis, especially in patients with pre-existing left ventricular impairment. Rich et al prospectively studied the occurrence of iatrogenic CHF in 401 patients hospitalised for $\mathrm{CHF}^{40} \mathrm{CHF}$ was considered iatrogenic in $28(7 \%)$ patients. As a result of the inability of the researchers to reliably assess causality between suspected inadequate medication and the onset of $\mathrm{CHF}$, most instances of iatrogenic $\mathrm{CHF}$ were attributed to excessive intravenous fluid administration. Therefore, the importance of inappropriate medication, such as withdrawal of ACE inhibitors, seems to have been underestimated in this study.

Patients with CHF deserve optimal treatment and investigation. However, some studies have reported flaws in the management of $\mathrm{CHF}$. Clarke et al carried out a retrospective study in six general practices of 505 patients given loop diuretics. ${ }^{41}$ Seventy four per cent of 281 patients who fulfilled diagnostic criteria for CHF were referred to hospital, but only one third had echocardiography. Furthermore, 234 of 281 patients who fulfilled diagnostic criteria for $\mathrm{CHF}$ were not treated with ACE inhibitors. Among them were 26 patients with documented evidence of left ventricular impairment. These findings strongly suggest shortcomings in the diagnosis and treatment of $\mathrm{CHF}$, but lack of information on the severity of $\mathrm{CHF}$ in this study makes it difficult to draw definite conclusions. Hillis et al reported in a review of the case notes of 343 patients discharged from hospital with a diagnosis of CHF that only $40 \%$ of patients received ACE inhibitors. ${ }^{42}$ Of patients with NYHA class III and IV CHF, only $50 \%$ were treated with ACE inhibitors at the time of discharge from hospital. Although retrospective studies may have methodological limitations, they clearly highlight some aspects of the management of $\mathrm{CHF}$ that need to be improved.

Prevention of iatrogenic CHF should be a major issue in optimising medical treatment in these patients.

\section{Conclusions}

Relapse in patients with $\mathrm{CHF}$ is the result of deteriorating underlying cardiac disease. Clinical practice and observational studies, however, have shown that precipitating factors can be identified in many patients with increased symptoms of CHF. These precipitating factors may have contributed to or even induced symptoms of CHF. Few studies have investigated these precipitating factors despite their presence in relapse of CHF. Suboptimal medical treatment and poor patient compliance in particular deserve more attention, as these factors can be considered as potentially preventable determinants of relapse of CHF.

Most of the discussed factors are common in daily practice, but little is known about their influence on cardiac performance in patients with CHF under everyday circumstances. Most studies on the haemodynamic effects of smoking, alcohol, or psychological stress have been laboratory experiments. Such findings do not always reflect the effects in clinical practice. Despite these limitations the negative haemodynamic effects of most precipitating factors discussed here are sufficient for them to be considered as unfavourable. Therefore, the presence of potential precipitating factors as listed in the table 1 should be routinely evaluated in patients presenting with CHF. Elimination of these precipitating factors, if possible, may contribute to the prevention of relapse in patients with $\mathrm{CHF}$.

1 Cowie MR, Mosterd A, Wood DA, et al. The epidemiology of heart failure. Eur Heart 7 1997; 18:208-25.

2 McGovern PG, Pankow JS, Shahar E, et al. Recent trends in acute coronary heart disease-mortality, morbidity, medical care, and risk factors. The Minnesota heart survey investigators. N Engl F Med 1996;334:884-90.

3 Gooding J, Jette AM. Hospital readmissions among the elderly. $\mathcal{F}$ Am Geriatr Soc 1985;33:595-601.

4 Vinson JM, Rich MW, Sperry JC, et al. Early readmission of elderly patients with congestive heart failure. $\mathcal{F} \mathrm{Am}$ Geriatr Soc 1990;38:1290-5.

5 Ghali JK, Kadakia S, Cooper R, et al. Precipitating factors leading to decompensation of heart failure. Traits among urban blacks. Arch Intern Med 1988;148:2013-16.

6 Opasich C, Febo O, Riccardi PG, et al. Concomitant factors of decompensation in chronic heart failure. Am $\mathcal{f}$ Cardiol of decompensation

7 Perlman LV, Ferguson S, Bergum K, et al. Precipitation of congestive heart failure: social and emotional factors. Ann Intern Med 1971;75:1-7.

8 Fabrizio L, Regan TJ. Alcoholic cardiomyopathy. Cardiovasc Drugs Ther 1994;8:89-94.

9 Dracup K, Baker DW, Dunbar SB, et al. Management of heart failure. II. Counseling, education, and lifestyle modifications. FAMA 1994;272:1442-6.

10 Molgaard H, Kristensen BO, Baandrup U. Importance of abstention from alcohol in alcoholic heart disease. Int $\mathcal{F}$ Cardiol 1990;26:373-5.

11 Pavan D, Nicolosi GL, Lestuzzi C, et al. Normalization of variables of left ventricular function in patients with alcoholic cardiomyopathy after cessation of excessive alcohol intake: an echocardiographic study. Eur Heart f 1987;8: 535-40.

12 Greenberg BH, Schutz R, Grunkemeier GL, et al. Acute effects of alcohol in patients with congestive heart failure. Ann Intern Med 1982;97:171-5.

13 Dargie HJ, McMurray JJ. Diagnosis and management of heart failure. BMF 1994;308:321-8.

14 Rabinowitz BD, Thorp K, Huber GL, et al. Acute hemodynamic effects of cigarette smoking in man assessed by systolic time intervals and echocardiography. Circulation 1979;60:752-60.

15 Goldbarg AN, Krone RJ, Resnekov L. Effects of cigarette smoking on hemodynamics at rest and during exercise. Normal subjects. Chest 1971;60:531-6.

16 Aronow WS, Cassidy J, Vangrow JS, et al. Effect of cigarette smoking and breathing carbon monoxide on cardiovascular hemodynamics in anginal patients. Circulation 1974;50: 340-7.

17 Pentecost B, Shillingford J. The acute effects of smoking on myocardial performance in patients with coronary arterial disease. Br Heart F 1964;26:422-9. 
18 Nicolozakes AW, Binkley PF, Leier CV. Hemodynamic effects of smoking in congestive heart failure. Am $7 \mathrm{Med} \mathrm{Sci}$ 1988;296:377-80

19 Giannuzzi P, Shabetai R, Imparato A, et al. Effects of mental exercise in patients with dilated cardiomyopathy and congestive heart failure. An echocardiographic Doppler study. Circulation 1991;83(suppl 4):II-155-65.

20 Rozanski A, Bairey CN, Krantz DS, et al. Mental stress and the induction of silent myocardial ischemia in patients with coronary artery disease. N Engl f Med 1988;318:1005-12.

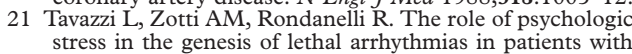
coronary artery disease. Eur Heart f 1986;7:99-106.

22 Tavazzi L, Zotti AM, Mazzuero G. Acute pulmonary edema provoked by psychologic stress. Report of two cases. Cardiology 1987;74:229-35.

23 Levy D, Larson MG, Vasan RS, et al. The progression from hypertension to congestive heart failure. $\mathcal{F} A M A$ 1996;275: hypertensior $1557-62$.

24 Visser FC. Congestive heart failure: pathophysiology and management with special reference to systemic hypermanagement with special reference to system
tension. F Cardiovasc Pharmacol 1991;18:S8-11.

25 Motz W, Vogt M, Scheler S, et al. Coronary circulation in arterial hypertension. If Cardiovasc Pharmacol 1991;17 S35-9.

26 Zanchetti A. Antihypertensive therapy: how to evaluate the benefits. Am F Cardiol 1997;79:3-8.

27 Middlekauff HR, Stevenson WG, Stevenson LW. Prognostic significance of atrial fibrillation in advanced heart failure. A study of 390 patients. Circulation 1991;84:40-8.

28 Carson PE, Johnson GR, Dunkman WB, et al. The influence of atrial fibrillation on prognosis in mild to moderate heart failure. The V-HeFT studies. The V-HeFT VA Cooperative Studies Group. Circulation 1993;87(suppl 6):VI102-10.

29 Lundstrom T, Karlsson O. Improved ventilatory response to exercise after cardioversion of chronic atrial fibrillation to exercise after cardioversion of chronic
sinus rhythm. Chest 1992;102:1017-22.

30 Kieny JR, Sacrez A, Facello A, et al. Increase in radionuclide left ventricular ejection fraction after cardioversion of chronic atrial fibrillation in idiopathic dilated cardiomyopathy. Eur Heart $\mathcal{F}$ 1992;13:1290-5.
31 Iga $\mathrm{K}$, Takahashi S, Yamashita $M$, et al. Reversible left venricular dysfunction secondary to rapid atrial fibrillation. nt $\mathcal{F}$ Cardiol 1993;41:59-64.

32 Grogan M, Smith HC, Gersh BJ, et al. Left ventricular dysfunction due to atrial fibrillation in patients initially believed to have idiopathic dilated cardiomyopathy. $A m \mathcal{F}$ Cardiol 1992;69:1570-3.

33 Rahimtoola SH. The hibernating myocardium in ischaemia and congestive heart failure. Eur Heart f 1993;14:22-6.

34 Unverferth DV, Magorien RD, Lewis RP, et al. The role of subendocardial ischemia in perpetuating myocardial failure in patients with nonischemic congestive cardiomyopathy. Am Heart f 1983;105:176-9.

35 Freeman LJ, Nixon PG, Sallabank P, et al. Psychological stress and silent myocardial ischemia. Am Heart $\mathcal{f}$ $1987 ; 114: 477-82$.

36 Tzivoni D. Effect of transient ischaemia on left ventricular function and prognosis. Eur Heart $\mathcal{F}$ 1993;14:2-7.

37 Carlson EB, Cowley MJ, Wolfgang TC, et al. Acute changes in global and regional rest left ventricular function after successful coronary angioplasty: comparative results in stable and unstable angina. I Am Coll Cardiol 1989;13: 1262-9.

38 Eraker SA, Kirscht JP, Becker MH. Understanding and improving patient compliance. Ann Intern Med 1984;100 258-68.

39 DiMatteo MR, Sherbourne CD, Hays RD, et al. Physicians' characteristics influence patients' adherence to medical the medical outcomes study. Health Psychol 1993;12:93-102.

40 Rich MW, Shah AS, Vinson JM, et al. Iatrogenic congestive heart failure in older adults: clinical course and prognosis. f Am Geriatr Soc 1996;44:638-43.

41 Clarke KW, Gray D, Hampton JR. Evidence of inadequate investigation and treatment of patients with heart failure. Br Heart f 1994;71:584-7.

42 Hillis GS, Trent RJ, Winton P, et al. Angiotensinconverting-enzyme inhibitors in the management of converting-enzyme inhibitors in the management of 1996;89:145-50. 